\title{
Pôles, polarisation et la diffusion du développement régional : notes de recherches
}

\author{
Jandir Ferrera de Lima, Ph.D. ${ }^{1}$ \\ Université Estadual do Oeste do Paraná (UNIOESTE - Brésil)
}

\section{INTRODUCTION}

\begin{abstract}
Les mouvements de décentrage et de recentrage dans l'espace économique nous ramènent à des études de la diffusion spatiale du développement économique au sein des régions.
\end{abstract}

Malgré que la science régionale soit récente, quelques recherches essayent de comprendre la nature de l'espace et les composantes du changement spatial ainsi que les impacts sur la localisation des secteurs économiques entre les régions. Pour comprendre la nature des changements spatiaux, il ne faut pas sous-estimer la réalité historique de la structuration des pôles. $\mathrm{La}$ localisation des pôles et leurs caractéristiques internes marquent les disparités géoéconomiques, qui représentent les formes que le développement économique va prendre spatialement.

Le développement économique survient dans une séquence de changements spatiaux dans la structure interne de l'économie et de la société régionale. Cette séquence de changements transformerait les économies retardataires en économies avancées: c'est-à-dire, des régions attachées à la production primaire s'orientent vers les secteurs secondaires et tertiaires.

Les disparités géoéconomiques surviennent au cours du processus de développement économique et de ses modifications spatiales. Elles résultent des forces de dispersion et de concentration. Un exemple est l'essor de l'économie de marché : $\mathrm{Au} \mathrm{XV} \mathrm{X}^{\mathrm{e}}$ siècle, celle-ci a relié des bourgs, des villes, des régions afin de pouvoir organiser et orienter la production. L'économie de marché va transformer toute l'Europe. Depuis que l'Europe s'est organisée dans les directives du marché, il est arrivé des disparités sur les gains de certaines régions par rapport à d'autres. Il y a des espaces dotés de conditions particulières à la transformation, comme l'Angleterre, la France et l'Allemagne, qui attirent de plus en plus d'activités de transformation. D'autres espaces demeurent faibles dans la transformation des ressources, comme l'Espagne, l'Italie, le Portugal. En fait, malgré ces disparités, l'Europe est une «économie - monde », c'est-à-dire dans la mesure où elle va former un ensemble économique. Cet ensemble possède une disparité géoéconomique : l'existence d'un pôle et d'une périphérie. En effet, il accepte toujours un pôle. Plus loin, il peut exister deux ou plusieurs pôles selon les caractéristiques de l'espace, mais ça n'est pas une constante. Au XVIII ${ }^{\mathrm{e}}$ siècle, par exemple, l'Europe possédait deux centres de décisions: Londres et Amsterdam. Au XX $\mathrm{XX}^{\mathrm{e}}$ siècle, Londres reste encore un centre très important. De la même façon, aux États-Unis, dans le XIX ${ }^{\mathrm{e}}$ siècle, les centres de décision étaient Washington et Boston. Actuellement, le centre le plus important est New York $^{2}$.

Les pôles coexistent avec d'autres centres moins importants. Il faut remarquer que ces événements de formation et de décadence des pôles ne laissent pas leur périphérie immobile. Les changements spatiaux conduisent toujours à des décentrages et recentrages. Dans un mouvement de recentrage, les pôles plus anciens perdront ainsi leurs pouvoirs de foyer émetteur et de foyer récepteur par rapport aux autres régions. Les pôles initialement atteints sont affaiblis au profit de zones rapprochées qui vont devenir plus ou moins, de façon provisoire, de nouvelles régions pôles ${ }^{3}$. 
Les mouvements de décentrage et de recentrage dans l'espace économique nous ramènent à des études de la diffusion spatiale du développement économique au sein des régions. Dans ce sens, il nous faudra définir deux concepts de base : le développement économique régional et la diffusion spatiale.

Le développement économique régional va entraîner des modifications, que ce soit dans la participation des secteurs, dans la composition du produit de l'économie ou encore dans la division sociale du travail entre les régions. En effet, cette dernière se rapporte à la spécialisation des régions et au partage de la main-d'œuvre entre les secteurs économiques.

Dans les secteurs, cette division représente aussi la spécialisation du travailleur dans les diverses étapes de la fabrication d'une marchandise. Mais, dans le contexte général du processus historique du développement économique, les secteurs secondaires et tertiaires auront de plus en plus d'importance par rapport au secteur primaire. La production agricole et l'élevage seront alors responsables d'une petite partie du Produit et de l'emploi, en opposition aux secteurs secondaires et tertiaires, qui attirent la majeure partie de ceuxci. Il en résulte une économie moins dépendante des zones rurales et une autre, qui va se retrouver plus dépendante des espaces urbanisés, parce que le secteur secondaire et le secteur tertiaire se retrouvent placés dans les villes. Ce changement dans les structures des régions se doit d'être accompagné par une amélioration du Produit par habitant.

Par rapport à cette conception du développement économique, et malgré le manque de données plus spécifiques et précises sur l'expansion de la production et de la population, nous pouvons observer qu'il y a une relation directe entre ces deux variables dans l'histoire des pays du tiers monde. L'accroissement ou le déclin de la population et de la production, demeurent toujours en évidence, dans la transformation des régions et des espaces des pays latino-américains, comme le Brésil, l'Argentine, le Pérou, le Mexique. Ils rapportent toujours des changements dans la façon d'exploiter les ressources, dans l'occupation des territoires et dans les possibilités de diversification ou de spécialisation des activités productives.

Quelques pays africains consacrent une grande partie de leur force de travail à la production primaire ou traditionnelle. D'autres pays, comme les États-Unis, le Canada et l'Allemagne, prennent la production secondaire (ou de transformation), comme la source principale de leur richesse. Parfois, la dynamique de quelques pays, comme les «paradis fiscaux» et les Antilles, se base sur la prestation des services ou des activités tertiaires, comme le tourisme.

La diffusion spatiale relève du phénomène de la propagation des hommes, des activités productives ou autres transformations majeures dans l'espace et dans le temps. Pour commencer un processus de diffusion, il doit y avoir l'existence d'une région pôle, même une ville, qui soit susceptible de jouer le rôle de «foyer émetteur ». Il devra aussi y avoir une périphérie capable d'adopter le potentiel des transformations économiques produites dans la diffusion. Le fait qu'une région progresse dans la division du travail est une référence à sa transformation productive.

\section{LES ÉLÉMENTS DE LA DIFFUSION SPATIALE}

La diffusion spatiale du développement économique régional entraîne cinq éléments :

1) Un espace.

2) Le temps : celui-ci se divise grâce à des intervalles successifs $(t, t+1, t+2, \ldots)$. Dans cet élément, l'introduction de l'échelle temporelle ou historique considère l'espace comme un système. Les transformations arrivent à partir d'une transformation spatiale antérieure. Donc, la notion d'espace est inséparable de l'idée de systèmes temporels. Par ce système, on comprend qu'il y a une succession de situations d'une population en état d'interaction permanente. Ces situations sont le résultat d'une situation précédente. Il faut qu'il y ait introduction de la dimension temporelle, et ce, dans l'étude de l'espace. 
3) L'élément diffuseur : L'élément-diffuseur peut être une innovation ou même une transformation plus générale, comme le processus de développement économique régional, caractérisé par le poids des secteurs et des activités productives plus modernes dans composition de l'économie régionale. L'étude de la localisation sectorielle, reliée à la composition de la production, est une référence du degré de développement économique des régions.

4) La localisation dans l'espace d'élément se diffusant au début et vers la fin d'une période. Dans ce cas, il faut mesurer le poids relatif de chaque région dans la localisation des secteurs économiques pendant ladite période. Ce qui déterminera la forme de la diffusion.
5) Les voies de mouvements: Comme la diffusion entraîne un changement spatial, il faut savoir quels composants ont été responsables du déplacement d'emploi ou du Produit entre les régions. Les voies de mouvement déterminent les places d'origine et du destin du changement. En effet, celles-ci précisent les régions qui ont eu plus de gains et les régions qui ont subi plus de pertes.

\section{La diffusion spatiale du développement économique régional entraîne cinq éléments.}

\section{LES FORMES DE LA DIFFUSION SPATIALE DU DÉVELOPPEMENT RÉGIONAL}

\section{Les formes de la diffusion se présentent} comme des résultats du changement spatial au niveau des régions et même des villes.

Tout le processus de développement entraîne toujours des disparités géo-spatiales ${ }^{4}$. Ces disparités résultent du processus de développement économique lui-même. Donc, à partir de la définition de la diffusion spatiale du développement économique, les régions se diviseront en deux groupes : le premier groupe sera marqué par le développement, par la localisation des secteurs modernes (secondaires et tertiaires). Le deuxième groupe est en retard et sous-développé, en raison d'une faible localisation des activités modernes ainsi qu'une forte localisation dans les activités primaires, par rapport aux régions développées. Même partagées, ces régions sont interactives et interdépendantes. Les formes de la diffusion se présentent comme des résultats du changement spatial au niveau des régions et même des villes. Ces formes sont par contiguïté, par percolation, par anisotropie, par hiérarchie urbaine et par migration.

La caractéristique principale de la diffusion spatiale par contiguité, c'est qu'elle se produit davantage dans les régions qui ont une frontière commune. Les régions les plus accolées au pôle sont passibles d'obtenir davantage d'associations et d'interactions que les régions plus éloignées. De ce fait, le processus de diffusion sera plus significatif dans les régions plus proches du foyer émetteur.

Dans la diffusion spatiale par percolation, le milieu n'est pas un foyer récepteur potentiel. Il ralentit le processus de développement du foyer émetteur (pôle) vers les régions périphériques. Dans la percolation, nous rencontrerons deux éléments : la composante structurelle, qui stimule la diffusion des activités modernes ainsi que la composante différentielle, qui est un ralentisseur à la diffusion. Parfois, la façon dont le milieu s'y prend pour modérer la diffusion est expliquée par diverses entraves à la mobilité des facteurs de production (travail, capital).

Dans la forme de la diffusion spatiale par axe ou par corridors, les effets de la diffusion du foyer émetteur sont plus forts dans les régions étant localisées dans un corridor. L'espace de liaison entre les pôles formera l'espace moteur entre cette association. Les pôles sont le foyer émetteur et récepteur potentiel du développement économique régional.

La diffusion spatiale par migration ou l'émergence des pôles remarque un mouvement de recentrage, les effets de la diffusion remplaceront les anciens pôles par d'autres. Les 
pôles plus anciens perdront ainsi leurs pouvoirs de foyer émetteur et de foyer récepteur par rapport aux autres régions. Dans un contexte historique, les pôles connaissent des moments d'expansion, d'accroissement et de déclin. L'histoire se base à partir d'émergence des pôles, parfois bien distribuée sur l'ensemble de l'espace, parfois non.

Au moment où l'émergence des pôles ne survient pas par hasard, mais de façon organisée dans un ensemble de villes, la forme de diffusion change par hiérarchie urbaine. Il y a deux visions différentes de la diffusion spatiale par hiérarchie urbaine: la première, à partir d'une hiérarchisation désordonnée et l'autre, d'une organisation urbaine tenue par des places centrales. Dans le cas de la première, la diffusion spatiale commence et se fait par les villes. Par contre, le processus de diffusion ne se fait pas avec une organisation spatiale précise. Il y aura des villes qui seront plus significatives au niveau des foyers récepteurs, d'autres qui seront plus dynamiques au niveau des foyers émetteurs, etc.

Dans une autre vision de la diffusion par hiérarchisation, le processus ne se fait pas de manière désordonnée. En effet, dans cette vision, la diffusion spatiale est affectée par la proximité des lieux, ainsi que par la position relative dans la région pouvant s'organiser dans des places centrales.

Dans le système des lieux centraux, l'idée de l'espace s'ouvre sur une nouvelle perspective : l'interaction entre les portions de l'espace. Cette interaction est produite par la gravitation à partir des portions de l'espace autour des centres (ou places centrales). Sur ces centres (ou places centrales), plusieurs zones de petites tailles vont former tout l'ensemble régional. La grande caractéristique des zones d'attraction est la présence de services tertiaires, produisant une hiérarchie dans la région ${ }^{5}$. La spécialisation à fournir des biens et des services, ainsi qu'un pouvoir d'attraction sur les petites villes définiront un centre. Les caractéristiques de cette attraction sont les suivantes : le transfert de la demande de la périphérie vers le centre, l'accroissement des investissements dans le centre, la migration des gens et des investissements vers le centre et la localisation des principales activités de commerce et de services dans le centre.

L'organisation des réseaux des villes sur l'espace sert à optimiser la proximité, les moyens de transport et la distribution de la population. Ces réseaux reflètent l'organisation ou la segmentation de l'espace, c'est-à-dire qu'ils réorganisent l'espace. Le processus de réorganisation d'espace va créer de nouvelles agglomérations, de nouveaux centres et de nouvelles aires de marché, basés sur une nouvelle conjoncture économique et potentielle de la production.

La diffusion par hiérarchie révèle qu'il y a tout un ensemble d'éléments responsables de l'organisation spatiale du développement économique régional. Elle deviendra un processus de réorganisation spatiale au sein de la localisation des activités productives aux villes.

\section{LE RETOUR À LA DIVISION SOCIALE DU TRAVAIL DANS LE PROCESSUS DE DÉVELOPPEMENT RÉGIONAL}

\section{À travers des études de la diffusion spatiale, il est possible d'examiner les changements et les transformations industrielles, productives, de localisation, etc.}

Le processus du développement économique régional est le résultat d'un changement spatial conduisant aux formes de la diffusion. Ce changement spatial possède des composantes structurales et différentielles. Il modifie la distribution sectorielle de l'emploi et de la production entre les différents secteurs économiques. Ainsi, il peut arriver des disparités régionales dans le poids des régions, par rapport à la localisation des activités productives. Mais ces changements n'arriveront pas à homogénéiser le territoire. Malgré un processus de déconcentration ou même de diffusion, il entraînera toujours une disparité géoéconomique caractérisée par des formes différentes de diffusion spatiale. 
À travers des études de la diffusion spatiale, il est possible d'examiner les changements et les transformations industrielles, productives, de localisation, etc. Dans les sciences humaines et sociales, la grande partie des approches analysent la diffusion spatiale comme étant un processus de dispersion des innovations ${ }^{6}$. Les innovations étudiées dans ces démarches se concentrent dans la diffusion des innovations particulières, comme les langues, les plantations agricoles, les nouvelles techniques, les animaux, les maladies, etc. En effet, il faut introduire le facteur temps et la division du travail dans l'analyse de la diffusion, principalement dans les études des régions du tiers monde. Dans les pays sousdéveloppés, l'avancée industrielle, et avec elles, les changements structuraux dans la division du travail, sont plus récents. Il y a eu des cas plus hétérogènes, au cours du développement, dans d'autres pays et régions. Par exemple, dans les pays développés, l'avancée de l'industrialisation a été plus longue et plus extensive; cette dernière ayant laissé des marques dans tous les espaces. Cependant, au tiers-monde, la diffusion a été plus courte et plus concentrée. Cette situation a demandé davantage d'études et d'analyses empiriques, dans des régions particulières des pays en développement.

\section{BIBLIOGRAPHIE ET NOTES}

${ }^{1}$ Braudel, Fernand. (1985). La dynamique $d u$ capitalisme, Paris, Arthaud.

${ }^{2}$ Christaller, Walter. (1966). Central Places in Southern Germany, (Traduction de l'original en allemand de 1933), New Jersey, Prentice-Hall.

${ }^{3}$ Dauphiné, André (1999). «Une théorie des disparités géographiques », Revue d'Économie Régionale et Urbaine (RERU), volume V, pp.899-914.

${ }^{4}$ PumainN, Denise; Saint-Julien, Thérèse. (2001). Les interactions spatiales, Paris, Armand Colin. 


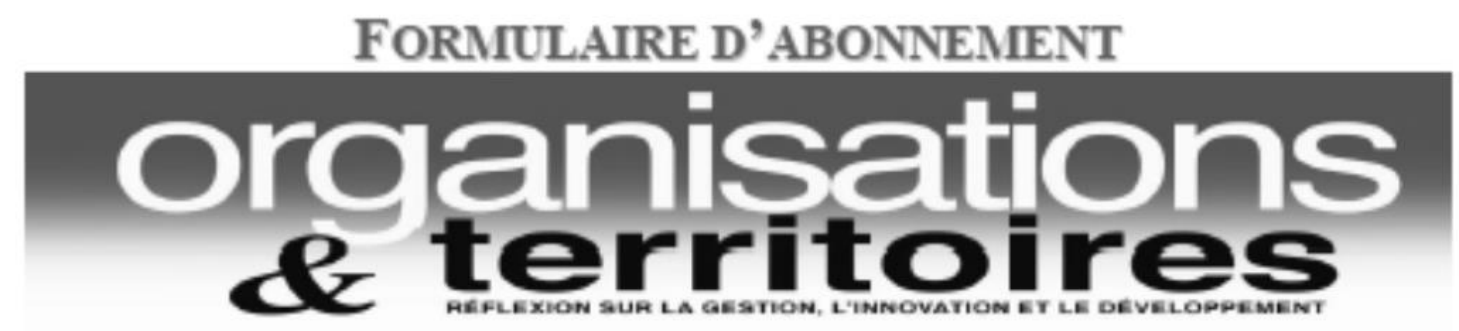

Je désire m'abonner à la revue Organisations et territoires et je joins un chèque au montant de : s

Nouvel abonné $(\sqrt{ }): \square$ Renouvellement $(\sqrt{ }): \square$ Date :
Abonnement institutionnel
(6 numéros)
$103,48 \$$
(Toutes taxes incluses)
Abonnement individuel
(6 numéros)
$66,69 \$$
(Toutes taxes incluses)
Abonnement étudiant
(6 numéros)
$48,29 \$$
(Tx inc) (Envoyer photocopie carte étud.)
Abonnement hors Canada
(6 numéros) $145,00 \$$
$(110 \$+35 \$$ frais de poste) (sans tows)

Tous les abonnements sont d'une durée de deux ans

- J'aimerais que mon abonnement débute avec le DERNIER numéro paru, soit :

Volume $\mathrm{N}^{\circ}$

- J'aimerais que mon abonnement débute avec le PROCHAIN numéro à paraître, soit :

Volume $\mathrm{N}^{\circ}$

Faites-nous parvenir vos coordonnées :

Nom :

Organisation :

Adresse:

\section{Téléphone :}

Telécopieur :

Courriel :

Adresse d'expédition :

Revue Organisations et territoires Université du Québec à Chicoutimi 555 , boulevard de l'Université, bureau H6-1360

Chicoutimi (Québec) CANADA G7H 2Bl

Teléphone : (418) 545-5011, poste 4530 Télécopieur : (418) 693-9072

Courriel : revueot@uqac.ca Site Web : www.uqac.ca/revueot 